SSC19-WKIV-02

\title{
HYTI: Thermal Hyperspectral Imaging From a Cubesat Platform
}

\author{
Robert Wright, Paul Lucey, Luke Flynn \\ 1. Hawaii Institute of Geophysics \& Planetology, University of Hawaii at Manoa \\ Honolulu, HI; 808-956-8760 \\ wright@higp.hawaii.edu \\ Miguel Nunes \\ 2. Hawaii Space Flight Laboratory, University of Hawaii at Manoa \\ Honolulu, HI; 808-956-0441 \\ manunes@hsfl.hawaii.edu \\ Thomas George \\ 3. SaraniaSat Inc. \\ Los Angeles, CA; 818-862-8960 \\ tom.george@saraniasat.com \\ Sarath Gunapala, David Ting, Sir Rafol \\ 4. Jet Propulsion Laboratory, California Institute of Technology. \\ Pasadena, CA; 818-393-4540 \\ sarath.d.gunapala@jpl.nasa.gov
}

\begin{abstract}
The HyTI (Hyperspectral Thermal Imager) mission, funded by NASA's Earth Science Technology Office InVEST (In-Space Validation of Earth Science Technologies) program, will demonstrate how high spectral and spatial longwave infrared image data can be acquired from a $6 \mathrm{U}$ CubeSat platform. The mission will use a spatially modulated interferometric imaging technique to produce spectro-radiometrically calibrated image cubes, with 25 channels between $8-10.7 \mu \mathrm{m}$, at a ground sample distance of $\sim 70 \mathrm{~m}$. The HyTI performance model indicates narrow band NE $\Delta$ Ts of $<0.3 \mathrm{~K}$. The small form factor of HyTI is made possible via the use of a no-moving-parts Fabry-Perot interferometer, and JPL's cryogenically-cooled BIRD FPA technology. Launch is scheduled for no earlier than October 2020. The value of HyTI to Earth scientists will be demonstrated via on-board processing of the raw instrument data to generate L1 and L2 products, with a focus on rapid delivery of precision agriculture metrics. .
\end{abstract}

\section{INTRODUCTION}

Since the launch of the Landsat 4 Thematic Mapper, scientists interested in studying the long-wave infrared (LWIR) thermal properties of Earth's surface, atmosphere, and water bodies at high-to-moderate resolution have been limited to making measurements at a $60-120 \mathrm{~m}$ ground sample (e.g. Landsat TM, ETM+), in no more than five spectral bands (e.g., Terra ASTER). This barely scratches the surface of the potential that the LWIR region of the spectrum has for quantifying Earth system processes. Operational acquisition of high spatial and spectral resolution LWIR data from Earth orbit would yield an hitherto unattainable measurement record for Earth scientists. Applications include mapping the chemistry of rocks and minerals exposed at Earth's surface ${ }^{1}$, the composition of volcanic gas and ash plumes ${ }^{2}$, and quantifying soil moisture content and evapotranspiration ${ }^{3}$.
The recently published Earth Science Decadal Survey ${ }^{4}$ explicitly identifies the provision of high spatial and either multi- or hyper-spectral thermal infrared data as a candidate measurement approach for achieving the Surface Biology and Geology Targeted Observable, and suggests that CubeSat constellations and alternative mission architectures be explored in order to provide, or complement, these important data. The HyTI mission is supportive of this recommendation, as it will demonstrate how recent innovations in LWIR imaging technologies can be combined to provide high spatial and spectral resolution LWIR image data from a $6 \mathrm{U}$ platform. 


\section{THE HYTI MISSION}

\section{The HyTI imager and measurement approach}

The HyTI imager is a novel, no-moving-parts hyperspectral imager that was originally developed using funding from DARPA and NASA. A prototype of the instrument (Fig. 1) has already been developed and flight tested in a light aircraft. Light from the scene is

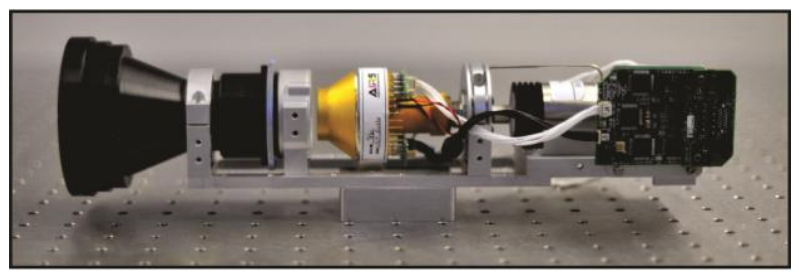

Figure 1: HyTI imager prototype

focused by a refractive lens and passed through a Fabry-Perot interferometer mounted directly above the focal plane array within the integrated dewar cooler assembly (IDCA). Forward motion of the platform allows interferograms of targets on the ground to be reconstructed, as each ground target is imaged at a succession of optical path differences as the fixed interference pattern is pushed along the ground in the in-track flight direction. Figure 2 illustrates the process.
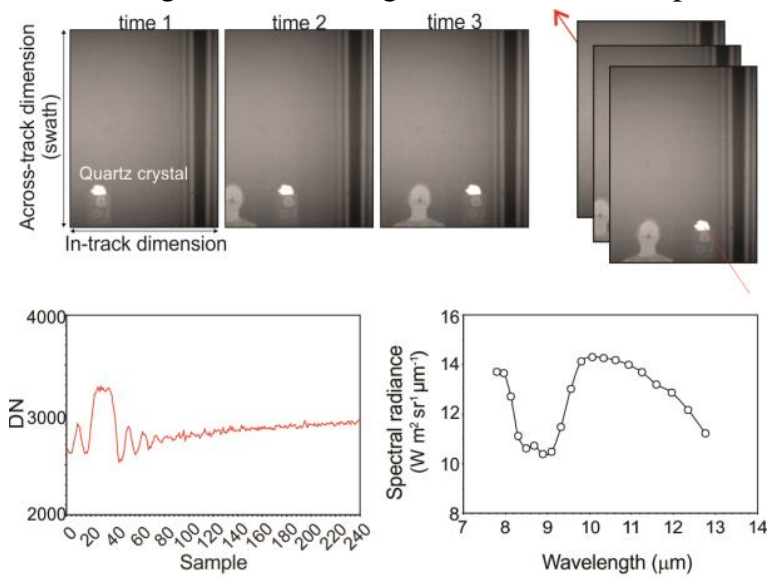

Figure 2: HyTI imaging approach

After co-registration of the image frames, standard Fourier Transform techniques ${ }^{5}$ are used to produce a spectro-radiometrically calibrated image cube. The Fabry-Perot interferometer consists of two pieces of germanium (AR coated), with a sloped air-gap between. Reflection, transmission and eventual recombination of rays that traverse this gap produces interference that can be sampled at the array. The slope ensures that optical path difference (hence fringe period, Fig. 2) varies linearly across the air gap (the broad dark vertical stripe in these images denotes the point at which the pieces of Ge are contacted, where no modulation takes place).
The spectral resolution of an interferometer is given by the ratio of the cut-off frequency to the number of samples in a single-sided interferogram ${ }^{6}$, and the fringe periodicity (number of samples) of HyTI is proportional to the design slope of the air-gap (although system fnumber provides an ultimate constraint on the spectral resolution achievable with this design ${ }^{7,8}$.
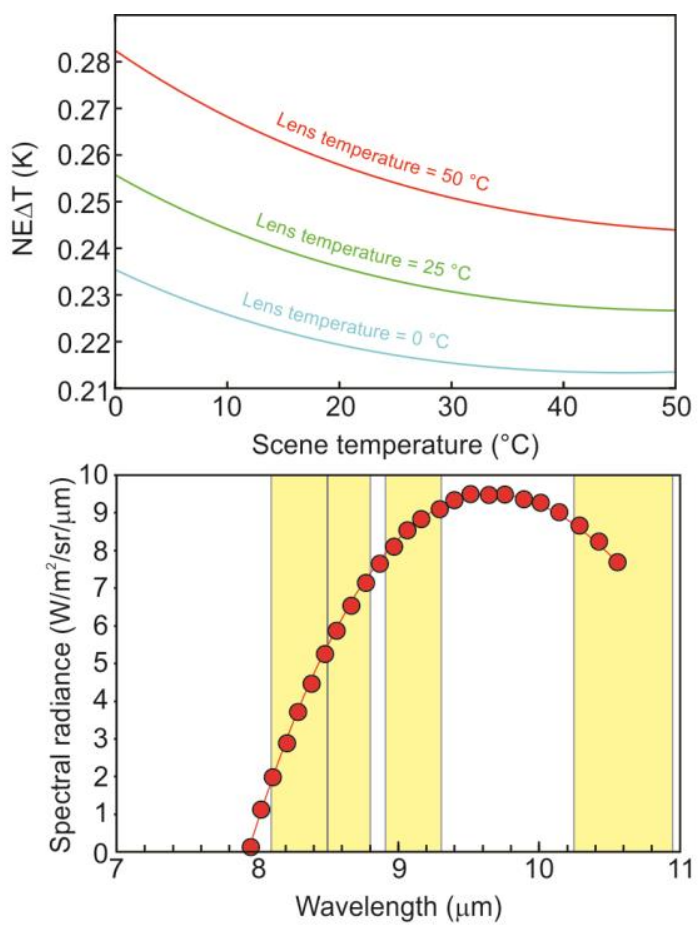

Figure 3: HyTI NEDT for several lens temperatures and scene temperatures (top) and band count (bottom). Yellow bars denote bandpasses of the Terra ASTER instrument

HyTI allows for high spatial and spectral resolution LWIR imaging by combining the multiplex advantage common to all interferometers with the sensitivity of JPL's Barrier Infra-Red Detector FPA technology ${ }^{9}$. Based on III-V compound semiconductors, the BIRD detectors offer a breakthrough solution for the realization of low cost (high yield), high-performance FPAs with excellent uniformity and pixel-to-pixel operability. These antimony ( $\mathrm{Sb}$ ) compound-based BIRD detectors outperform existing TIR detectors including Quantum Well Infrared Photodetectors (QWIPs). To achieve acceptable dark current levels (see below), the FPA must be maintained at a temperature of $68 \mathrm{~K}$. The spectral sensitivity is in the range 8-10.7 $\mu \mathrm{m}$, with a quantum efficiency of $35 \%$. For HyTI an FPA of $320 \times 512$ elements will be used, with 320 detectors used to define the field of view (swath). Not all 640 detectors are required to sample the interference pattern generated by the Fabry-Perot 
interferometer, and HyTI will window the array, reading out frames of $512 \times 320$ at $160 \mathrm{~Hz}$. This frame rate allows for oversampling in the in-track dimension at orbital velocity.

From an assumed orbital altitude of $\sim 400 \mathrm{~km}$ (i.e., ISS orbit the design ground sample distance of HyTI will be $\sim 70 \mathrm{~m}$. To achieve this a multi-element refractive lens will be used, with an IFOV of $0.15 \mathrm{mrad}$, and an fnumber of 3.44 (240 mm focal length). 25 spectral samples between 8-10.7 $\mu \mathrm{m}$ will be acquired (spectral resolution of $13 \mathrm{~cm}^{-1}$ ). Our performance model (Fig. 3) indicates that NE $\Delta$ Ts of $<0.3 \mathrm{~K}$ are attainable (at this spectral resolution), for source temperatures in the range $0-50{ }^{\circ} \mathrm{C}$.

\section{SPACECRAFT}

InVEST is targeted towards platforms exclusively in the $1 \mathrm{U}$ to $6 \mathrm{U}$ form-factor. HyTI will use a $6 \mathrm{U}$ bus. Figure 4 shows a conceptual rendering of the HyTI spacecraft. It is anticipated that $3.5 \mathrm{U}$ to $4 \mathrm{U}$ of the $6 \mathrm{U}$
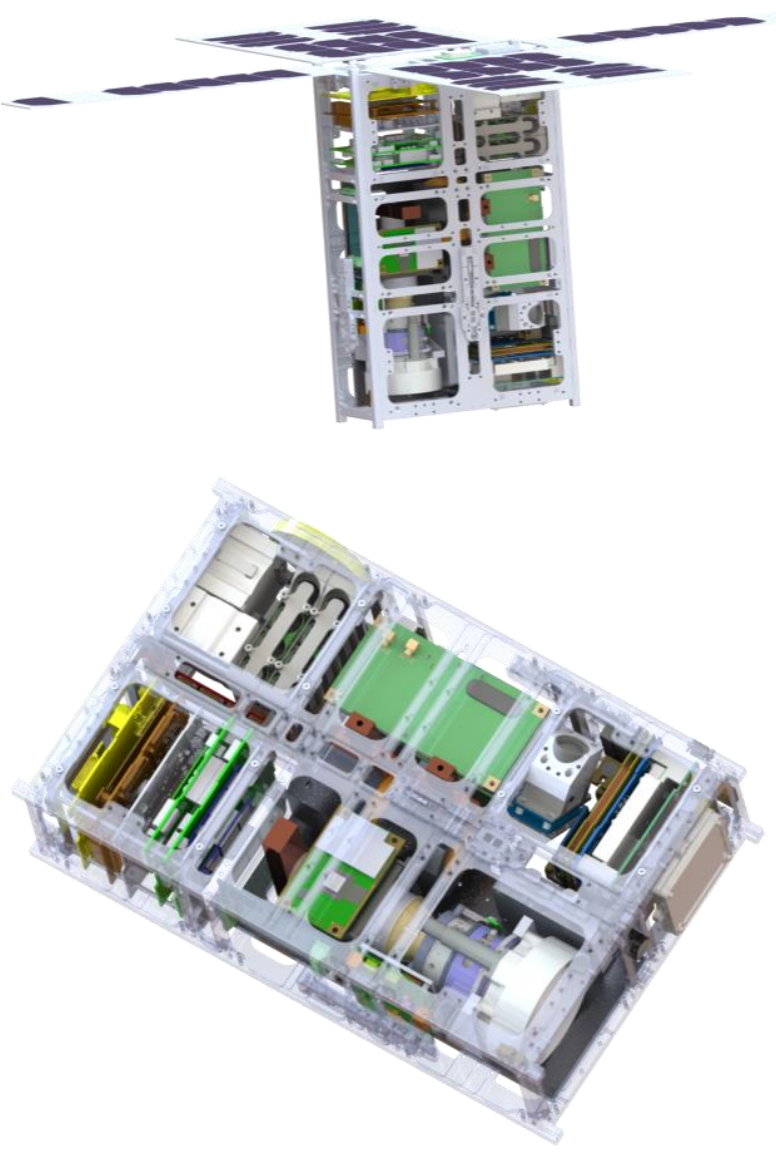

Figure 4. Rendering of HyTI $6 \mathrm{U}$ bus available will be required for the payload, which includes the IDCA (provided by American Infrared Solutions; https://www.go-airs.com/), the cryocooler (AIM SF070; https://www.aim-ir.com), the multielement refractive lens (provided by New England Optical Systems; http://www.neos-inc.com/), and the payload on-board computer (Unibap Deep Delphi iX5; https://unibap.com/). The Fabry-Perot interferometer will be provided by LightMachinery (https://lightmachinery.com/). ISIS (Innovative Solutions In Space) are our partner for the 6U bus. Communication will be via $\mathrm{X}$-band for downlink (Sband redundant) with $\mathrm{S}$-band up ( $\mathrm{S}$-band redundant), as well as a Globalstar transmit/receive capability.

\section{MISSION OPERATIONS AND SCIENCE FOCUS}

HyTI is a technology demonstration, not a science mission. Nevertheless, to demonstrate the applicability of HyTI's innovative technologies to making Earth science measurements, a mission must be defined. The science focus of HyTI is precision agriculture. Developing a detailed understanding of the movement, distribution and availability of water is a critical measurement objective for NASA's, as described in the recently published "Thriving on Our Changing Planet: A Decadal Strategy for Earth Observation from Space"4. HyTI will be designed to 1. Map irrigated and rain-fed cropland areas; 2. Determine crop water use (actual evapotranspiration) of major world crops; 3. Establish crop water productivity ("crop per drop").

The HyTI data rate is high, requiring onboard processing of the raw frames to the final Level 1 calibrated radiance cubes (i.e., Figure 2); this achieves a $\times 10$ reduction in data volumes even before lossless (3:1) compression. To achieve this, HyTI will demonstrate onboard processing from L0 to L1 (i.e. frame-to-frame co-registration, FFT and spectral calibration) using the Unibap Deep Delphi iX5 heterogeneous computer platform, which offers CPU, GPU and FPGA capability. SaraniaSat's weak signal detection algorithms will also be implemented on the Unibap DD $\mathrm{iX} 5$, to demonstrate derivation of L2 products onboard HyTI, and low latency delivery of those products to stake-holders.

Current mission operations is to image whenever HyTI is over the lower 48 states of the USA. This equates to HyTI acquiring data on five to seven orbits per day, for an average of six minutes per pass. We estimate that this raw data can be processed to calibrated radiance cubes in a further two orbits, with subsequent downlink (via KSAT) to a receiving station in Spain. Data collection, data processing, and data transmission 
cannot occur simultaneously due to the power constraints imposed by the $6 \mathrm{U}$ bus. Figure 5 shows a day-in-the-life simulation for HyTI which shows that the $40 \mathrm{~W}$ available via the four deployable solar panels (Fig. 3) and the 87 Wh battery allows this mission to be achieved.

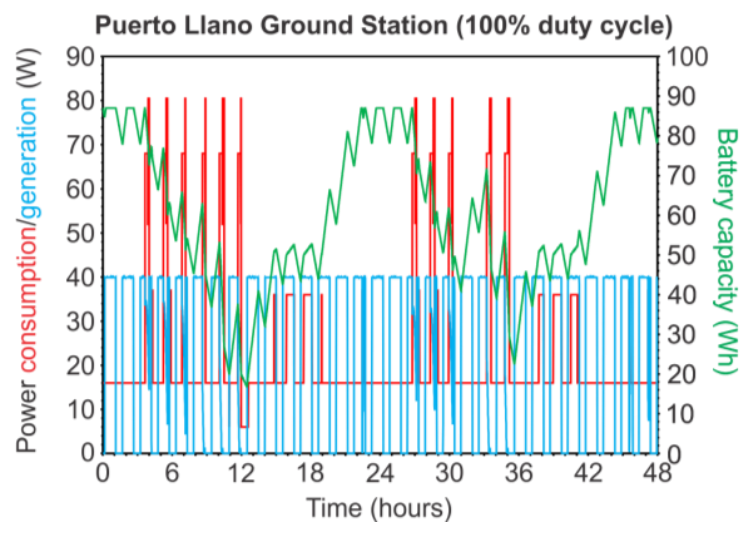

Figure 5. Day-in-the-life power budget for HyTI baseline mission

A proposal has been accepted for HyTI to the NASA CubeSat Launch Initiative. As HyTI is a thermal infrared imager, either a traditional near-Polar, sunsynchronous or an inclined (e.g. ISS) orbit can be utilized for the mission.

\section{CONCLUSIONS}

The HyTI (Hyperspectral Thermal Imager) mission will demonstrate how high spectral and spatial long-wave infrared image data can be acquired from a $6 \mathrm{U}$ CubeSat platform. The mission will use a spatially modulated interferometric imaging technique to produce spectroradiometrically calibrated image cubes, with 25 channels between 8-10.7 $\mu \mathrm{m}$, at a ground sample distance of $\sim 70 \mathrm{~m}$. The HyTI performance model indicates narrow band $\mathrm{NE} \Delta \mathrm{Ts}$ of $<0.3 \mathrm{~K}$. The small form factor of HyTI is made possible via the use of a no-moving-parts Fabry-Perot interferometer, and JPL's cryogenically-cooled BIRD FPA technology. Launch is scheduled for no earlier than November 2020.

\section{Acknowledgments}

HyTI is funded by NASA's Earth Science Technology Office InVEST (In-Space Validation of Earth Science Technologies) program element (grant number 80NSSC18K1601). The research was partly carried out at the Jet Propulsion Laboratory, California Institute of Technology, under a contract with the National Aeronautics and Space Administration.

\section{References}

1. J. L. Hall, J. A. Hackwell, D. M. Tratt, D. W. Warren, and S.J Young, "Space-based mineral and gas identification using a high-performance thermal infrared imaging spectrometer", Proc. SPIE 7082, 70820M, 2008

2. A. Gabrieli, J. Porter, R. Wright, and P. Lucey, P, "Validating the accuracy of $\mathrm{SO}_{2}$ gas retrievals in the thermal infrared $(8-14 \mu \mathrm{m})$," Bull. Volcanol., 80, https://doi.oeg/10.1007/s00445-017-1172-2, 2017.

3. M. C. Anderson, J. M. Norman, W. P. Kustas, R. Houborg, P. J. Starks, and N. Agam, "A thermalbased remote sensing technique for routine mapping of land-surface carbon, water and energy fluxes from field to regional scales," Remote. Sens. Environ., 112 (12), 4227-4241, 2008.

4. National Academies of Sciences, Engineering, and Medicine, "Thriving on Our Changing Planet A Decadal Strategy for Earth Observation from Space," The National Academies Press. https://doi.org/10.17226/24938, 2018.

5. L. Mertz, “Transformations in Optics," Wiley, 1965.

6. P.R. Griffiths and J.A. DeHaseth, "Fourier Transform Infrared Spectrometry," Wiley, 1986

7. P. G., Lucey, and J. Akagi, "A Fabry-Perot interferometer with a spatially variable resonance gap employed as a Fourier transform spectrometer," Proc. SPIE 8048, 80480K-1, 2011.

8. P.G. Lucey, J.L., Hinrichs, and J. Akagi, "A compact LWIR hyperspectral system employing a microbolometer array and a variable gap FabryPerot interferometer employed as a Fourier transform spectrometer," Proc. SPIE 8390, 83900R-1, 2012.

9. A. Ting, A. Soibel, A. Khoshakhlagh, S. B. Rafol, S. A. Keo, L. Höglund, A. M. Fisher, E. M. Luong, and S. D. Gunapala, "Mid-wavelength high operating temperature barrier infrared detector and focal plane array," Appl. Phys. Lett. 113, 021101, 2018. 